\title{
Long non-coding RNA BRM promotes proliferation and invasion of papillary thyroid carcinoma by regulating the microRNA-331-3p/SLC25A1 axis
}

\author{
SHIHONG LIU ${ }^{1}$, DEPING ZHANG ${ }^{1}$, LI CHEN $^{1}$, SHANGFANG GAO $^{1}$ and XIU HUANG ${ }^{2}$ \\ Departments of ${ }^{1}$ Nuclear Medicine and ${ }^{2}$ Radiography, The People's Hospital of Tong Liang District, \\ Chongqing 402560, P.R. China
}

Received January 23, 2019; Accepted August 30, 2019

DOI: $10.3892 / \mathrm{ol} .2020 .11418$

\begin{abstract}
Long non-coding RNA BRM (lncBRM) was first identified in liver cancer stem cells and was reported to promote multiple cancer types. However, the function of IncBRM in papillary thyroid carcinoma (PTC) remains unclear. The primary focus of the present study was to determine the biological role of IncBRM in PTC. Reverse transcription-quantitative PCR assays revealed that IncBRM was upregulated in PTC tissues and cells. Cell Counting Kit-8, Transwell invasion and colony-formation assays were performed to assess cell proliferation, invasion and migration, respectively. Furthermore, high expression of lncBRM was associated with poor overall survival time in patients with PTC. IncBRM knockout significantly suppressed cell proliferation, migration and invasion. IncBRM was predicted to bind to microRNA (miR)-331-3p and targets SLC25A1. Overexpression of miR-331-3p or inhibition of SLC25A1 resulted in significantly suppressed proliferation, migration and invasion of PTC cells. Rescue assays demonstrated that inhibition of miR-331-3p significantly abrogated the effects of lncBRM knockout on PTC cell proliferation, migration and invasion. In conclusion, the present study suggests that lncBRM promotes PTC by regulating miR-331-3p and targeting SLC25A1.
\end{abstract}

\section{Introduction}

In 2018, thyroid cancer was the most common thyroid malignancy, accounting for $\sim 1 \%$ of all malignant tumors, and includes papillary, follicular, undifferentiated and medullary carcinomas (1). Thyroid cancer occurs mostly in

Correspondence to: Dr Xiu Huang, Department of Radiography, The People's Hospital of Tong Liang District, 528 Zhongxing East Road, Dongcheng Street, Chongqing 402560, P.R. China

E-mail: xiuhuang111@sina.com

Key words: long non-coding RNA BRM, micoRNA-331-3p, SLC25A1, papillary thyroid carcinoma young adults aged $\sim 40$ years, and the ratio men to women is $1.0: 2.5$ to $1.0: 3.0$ (2). Papillary thyroid carcinoma is typically exhibits lower malignancy, and better prognosis and is the most common type of thyroid cancer, accounting for $85-90 \%$ of cases (3). In recent years, the incidence of thyroid cancer has steadily increased (4). Most patients ( 90\%) with PTC can be effectively treated via surgical removal and have a good 5-year survival rate (5). However, a small proportion of patients with PTC have poor prognosis and survival rates (6). Therefore, it is necessary to illustrate the molecular mechanism underlying proliferation and invasion of thyroid cancer, in order to identify potential therapeutic targets (7).

Long non-coding RNA (lncRNAs) is a type of RNA $>200$ nucleotides in length, with little or no coding potential (8). Recently, lncRNAs were found to serve a pivotal role in several biological processes (9-11), such as embryo development $(12,13)$, immunology $(14,15)$ and cancer (16-18). Dysregulation of IncRNAs have been shown to be involved in tumorigenesis and tumor progression in PTC (19-21), suggesting the potential of lncRNAs as diagnostic markers or therapeutic agents for PTC $(22,23)$. For example, IncRNA LINC00460 promotes carcinogenesis by sponging microRNA (miR)-613 in PTC and upregulating the expression of sphingosine kinase 2 (24). Additionally, lncRNA FOXD2-AS1 was found to be upregulated in PTC tissues and function as a competing endogenous RNA (ceRNA) to enhance the expression of KLK7, by sponging miR-485-5p (25).

lncBRM was first reported to be highly expressed in liver stem cells and was shown to be associated with the progression of liver cancer, by interacting with BRM and regulating the YAP signaling pathway (26). Moreover, IncBRM was proven to exhibit a pivotal role in ovarian cancer, through the upregulation of SOX4 and thereby facilitating proliferation, migration and invasion of ovarian cancer cells (27). Furthermore, IncBRM was shown to regulate the proliferation and invasion of colorectal cancer cells, by sponging miR-204-3p and upregulating translationally-controlled tumor protein 1 (TPT1) (28). However, the role of lncBRM in PTC remains unclear. In the present study, the expression patterns, biological functions and mechanisms of action of IncBRM in PTC progression were elucidated. 


\section{Materials and methods}

Samples and cell lines.PTC samples and matched normal tissues were obtained from 90 patients (mean age, $63.5 \pm 5.1$ years; age range, 37.5-72.4 years; 32 men and 58 women) with PTC undergoing surgery at The People's Hospital of Tong Liang District (Chongqing, China). The pathological diagnosis of all specimens was graded according to the classification of thyroid malignancy of the World Health Organization (2004) by two experienced pathologists (29). Among these patients, 41 cases were high-grade and 41 cases were low-grade, and 46 cases were non-metastasis and 44 cases were metastasis. All tissues were frozen in liquid nitrogen until further use. The study was approved by the Ethics Committee of The People's Hospital of Tong Liang District (permission no. 2015KT57). All patients provided written informed consent.

The normal human thyroid follicular epithelial cell line Nthy-ori 3-1, and human thyroid cancer cell lines TPC-1 and SW1736, were obtained from the American Type Culture Collection. All cells were cultured in RPMI-1640 medium (Thermo Fisher Scientific, Inc.) supplemented with 10\% FBS (HyClone; GE Healthcare Life Sciences), $100 \mathrm{U} / \mathrm{ml}$ penicillin and $100 \mu \mathrm{g} / \mathrm{ml}$ streptomycin (both from Sigma-Aldrich; Merck $\mathrm{KGaA}$ ) at $37^{\circ} \mathrm{C}$ in $5 \% \mathrm{CO}_{2}$.

Plasmids and transfections. TPC-1 and SW1736 cells were seeded into six-well plates at a density of $1 \times 10^{5}$ cells/well, and cultured overnight until they reach $70-80 \%$ confluence. miR-331-3p mimic, empty vector control, miR-331-3p inhibitor were used at $50 \mathrm{nM}$. The short hairpin (sh)RNA specifically targeting lncBRM, SLC25A1 and scrambled negative control shRNA were provided by GenePharma Co. Ltd and used at $1 \mathrm{mg} / \mathrm{ml}$. The coding sequence of SLC25A1 was constructed into pMy vectors (Addgene, Inc.) to overexpress SLC25A1 that was used at $1 \mathrm{mg} / \mathrm{ml}$. The empty control, which served as a negative control, was also used at $1 \mathrm{mg} / \mathrm{ml}$. Plasmids were transfected into the cells using Lipofectamine ${ }^{\mathrm{TM}} 2000$ (Invitrogen; Thermo Fisher Scientific, Inc.), according to the manufacturer's instructions. After $6 \mathrm{~h}$, successfully transfected cells were confirmed by reverse transcription-quantitative PCR (RT-qPCR) analysis and cultured in six-well plates using RPMI-1640 medium containing $10 \% \mathrm{FBS}$ for two days at $37^{\circ} \mathrm{C}$ and $5 \% \mathrm{CO}_{2}$ to expand for subsequent experiments. The sequences of the primers used were as follows: miR-331-3p mimics, 5'-GCCCCUGGGCCUAUCCUAGAA-3'; and miR-331-3p inhibitor, 5'-TTCTUGGUTUGGCCCUGG GGC-3'. pMIR-SLC25A1-3'UTR (wild type or mutant) or pMIR-IncBRM (wild type or mutant) and miR-331-3p mimics were transfected into TPC-1 or SW1736 cell lines along with pRL-TK vectors (Promega Corporation). Following culture for $24 \mathrm{~h}$, luciferase activity was measured using a dual $\mathrm{Glo}^{\mathrm{TM}}$ Luciferase assay system (Promega Corporation) according to the manufacturer's protocols and normalized to Renilla luciferase activity.

$R N A$ preparation and $R T-q P C R$. Total RNA was isolated from tissues or cells using TRIzol ${ }^{\circledR}$ reagent (Invitrogen; Thermo Fisher Scientific, Inc.) according to the manufacturer's instructions. Total RNA was subsequently reverse-transcribed into cDNA using the PrimeScript RT reagent kit (Promega
Table I. Associations between lncBRM expression and clinicopathological features in 90 patients with papillary thyroid carcinoma.

\begin{tabular}{lccc}
\hline Variables & $\begin{array}{c}\text { Low } \\
(\mathrm{n}=45)\end{array}$ & $\begin{array}{c}\text { High } \\
(\mathrm{n}=45)\end{array}$ & P-value \\
\hline $\begin{array}{l}\text { Age, years } \\
>60\end{array}$ & 23 & 18 & 0.397 \\
$\leq 60$ & 22 & 27 & \\
Sex & & & 0.833 \\
$\quad$ Male & 21 & 23 & \\
$\quad$ Female & 24 & 22 & \\
$\quad$ TNM stage & & & 0.033 \\
I-II & 30 & 19 & \\
$\quad$ III-IV & 15 & 26 & \\
Lymph node metastasis & & & 0.020 \\
$\quad$ Yes & 16 & 28 & \\
No & 29 & 17 & \\
\hline
\end{tabular}

TNM, Tumor-Node-Metastasis.

Corporation) according to the manufacturer's protocol. The reaction was performed at $42^{\circ} \mathrm{C}$ for $1 \mathrm{~h}$, and the enzyme was subsequently inactivated at $85^{\circ} \mathrm{C}$ for $5 \mathrm{~min}$. qPCR was performed using SYBR Green PCR Master mix reagents (Takara Bio, Inc.) in a 7300 Real-Time PCR system (Applied Biosystems; Thermo Fisher Scientific, Inc.). miR-331-3p expression was determined using SYBR Premix Ex Taq II (GeneCopoeia, Inc.) according to the following conditions: $10 \mathrm{~min}$ of pre-denaturation at $95^{\circ} \mathrm{C}$, followed by 40 cycles of $10 \mathrm{sec}$ denaturation at $95^{\circ} \mathrm{C}, 20 \mathrm{sec}$ annealing at $60^{\circ} \mathrm{C}$ and $30 \mathrm{sec}$ extension at $72^{\circ} \mathrm{C}$. SLC25A1 mRNA expression was measured using a SYBR Green PCR Master Mix (Applied Biosystems; Thermo Fisher Scientific, Inc.) with the following procedures: $10 \mathrm{~min}$ pre-denaturation at $95^{\circ} \mathrm{C}$, followed by 36 cycles of $10 \mathrm{sec}$ denaturation at $95^{\circ} \mathrm{C}, 20 \mathrm{sec}$ annealing at $60^{\circ} \mathrm{C}$ and $34 \mathrm{sec}$ extension at $72^{\circ} \mathrm{C}$. $18 \mathrm{~S}$ was used as internal reference for miR-331-3p and SLC25A1 mRNA expression. Relative expression of miR-6852 and LEF1 mRNA was calculated using $2^{-\Delta \Delta \mathrm{Cq}}$ method (30). The sequences of the primers used were as follows: SLC25A1 forward, 5'-CCG TCAGGTTTGGAATGTTCG-3' and reverse, 5'-TAACCC CGTGGAAGAATCCTC-3'; IncBRM forward, 5'-GGTCAA GAGGCCAGGAAGAG-3' and reverse, 5'-TTCTCACTT CAGCCCAATGCT-3'; and 18S forward, 5'-CAGCCACCC GAGATTGAGCA-3' and reverse, 5'-TAGTAGCGACGGGCG GTGTG-3'.

Cell counting Kit-8 (CCK-8) assays. TPC-1 and SW 1736 cells $\left(1 \times 10^{4}\right)$ were seeded in a 96-well plate and cultured in RPMI-1640 medium supplemented with $10 \%$ FBS at $37^{\circ} \mathrm{C}$ and $5 \% \mathrm{CO}_{2}$. CCK-8 solution (10 $\mu \mathrm{l}$; Beyotime Institute of Biotechnology) was added into each well for $4 \mathrm{~h}$ at $37^{\circ} \mathrm{C}$. Cell proliferation was assessed 24,48 and $72 \mathrm{~h}$ post-transfection. The absorbance was measured at $450 \mathrm{~nm}$ using a microplate reader (BioTek Instruments, Inc.). 
A
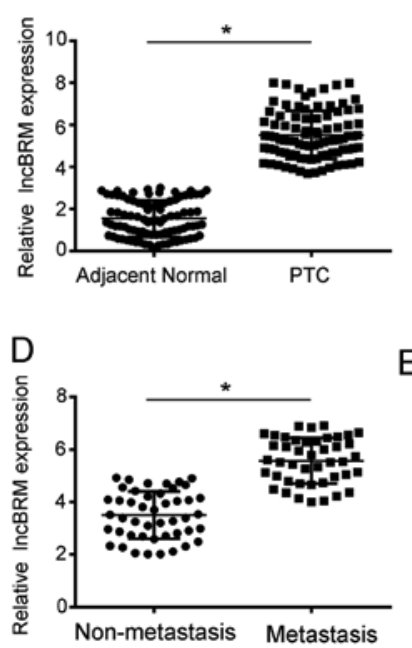

B

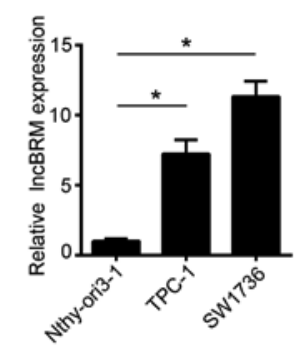

E

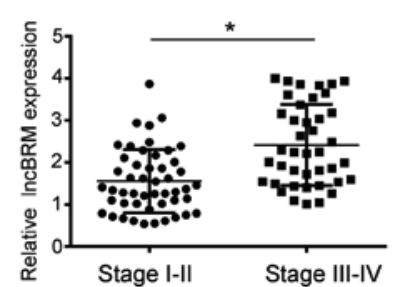

C

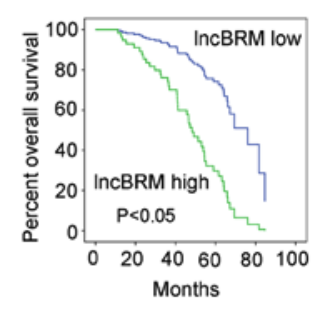

Figure 1. IncBRM is upregulated in PTC tissues and cell lines and is associated with prognosis. (A) RT-qPCR was performed to examine the expression level of lncBRM in 90 pairs of PTC tissues and adjacent normal tissues. (B) RT-qPCR was performed to examine the relative expression levels of lncBRM in normal Nthy-ori3-1 cell lines and TPC-1 and SW1736 PTC cell lines. (C) Patients with PTC were divided into two groups, lncBRM high level (n=45) and lncBRM low level ( $\mathrm{n}=45)$, to perform Kaplan-Meier analysis of overall survival time, followed by a log-rank test. (D) The expression levels of lncBRM in PTC tissues with metastasis $(n=45)$ or no metastasis $(n=45)$ were examined by RT-qPCR. (E) Expression levels of lncBRM in stage I-II ( $n=45)$ or stage III-IV ( $=45)$ PTC tissues were determined using RT-qPCR. Fold changes were normalized to $18 \mathrm{~S}$ in all cases. ${ }^{*} \mathrm{P}<0.05$. RT-qPCR, revere transcription-quantitative PCR; lnc, long non-coding; PTC, papillary thyroid carcinoma.

Colony-formation assays. Cells in the logarithmic growth phase were treated with $0.25 \%$ trypsin to give single-cell suspensions. Each group was inoculated at a density of $\sim 1,000$ cells/well in the culture medium. The culture was terminated when colonies were visible, after 2-3 weeks. The supernatant was discarded and the colonies were fixed at room temperature with $4 \%$ paraformaldehyde for $15 \mathrm{~min}$ and stained with $0.5 \%$ Giemsa for $30 \mathrm{~min}$ at room temperature. The number of colonies were examined under a light microscope (magnification, $\mathrm{x} 100$ ).

Bioinformatics analysis. miR-331-3p was predicted as a potential lncBRM target by using miRDB tool (http://mirdb. org/miRDB/index.html). miRDB is an online database for miRNA target prediction and functional annotations. All the targets in miRDB were predicted by a bioinformatics tool, MirTarget, which was developed by analyzing thousands of miRNA-target interactions from high-throughput sequencing experiments. SLC25A1 was predicted as a potential miR-331-3p target by using TargetScan7 tool (http://www.targetscan.org/vert_72/). TargetScan predicts biological targets of miRNAs by searching for the presence of conserved $8 \mathrm{mer}, 7 \mathrm{mer}$, and $6 \mathrm{mer}$ sites that match the seed region of each miRNA.

Transwell experiments. Two days after transfection, TPC-1 and SW1736 cells were prepared as single cell suspensions $\left(1 \times 10^{5}\right.$ cells $\left./ \mathrm{ml}\right)$ in serum-free RPMI-1640. Transwell chambers ( $8 \mathrm{~mm}$ pore; EMD Millipore) were inserted into 24 -well plates containing $600 \mu 1$ RPMI-1640 supplemented with 10\% FBS in the lower chamber. A $100 \mu \mathrm{l}$ cell suspension containing $1 \times 10^{4}$ cell was added into the upper chamber. Cells were cultured for $48 \mathrm{~h}$ at $37^{\circ} \mathrm{C}$. Non-migrated cells were scraped off and migrated cells were fixed using $4 \%$ formaldehyde for $30 \mathrm{~min}$ at room temperature, stained using $0.5 \%$ crystal violet for $30 \mathrm{~min}$ at room temperature and counted under a light microscope (magnification, x200). The aforementioned procedure was also used to detect cell invasion; however, for the cell invasion assay, Transwell were pre-coated with $100 \mathrm{ml}$ Matrigel $\left(1 \mathrm{mg} / \mathrm{ml}\right.$; BD Biosciences) for $30 \mathrm{~min}$ at $37^{\circ} \mathrm{C}$, prior to cell seeding.

Western blot analysis. Total proteins in each cell sample were extracted using RIPA lysis buffer (Beyotime Institute of Biotechnology). Proteins were quantified using a bicinchoninic acid kit (Pierce; Thermo Fisher Scientific, Inc.), and $40 \mathrm{mg}$ protein/lane were separated by $10 \%$ SDS-PAGE. Proteins were then transferred onto polyvinylidene difluoride membranes. Membranes were subsequently incubated with 5\% skimmed milk for $1 \mathrm{~h}$ at room temperature. TBS supplemented with $0.1 \%$ Tween 20 (TBST) was used to wash the membrane. Membranes were incubated with primary antibodies against SLC25A1 (1:2,000; cat. no. 15235-1-AP; ProteinTech Group, Inc.) and $\beta$-actin (1:5,000; cat. no. 60008-1-Ig; ProteinTech Group, Inc.) for $12 \mathrm{~h}$ at $4^{\circ} \mathrm{C}$. Membranes were then incubated with goat anti-mouse (cat. no. 70-GAM007) and goat anti-rabbit (cat. no. 70-GAR007) secondary antibodies [1:5,000; Multisciences (Lianke) Biotech Co., Ltd.] for $2 \mathrm{~h}$ at room temperature. Membranes were washed with three times TBST for $15 \mathrm{~min}$. Protein expression was monitored using the Pierce $^{\mathrm{TM}}$ ECL Western Blotting Substrate (Beijing Solarbio Science \& Technology Co., Ltd.) and quantified using Quantity One software v4.62 (Bio-Rad Laboratories Inc.).

Statistical analysis. GraphPad Prism version 6 (GraphPad Software, Inc.) was used to analyze the data. All results are expressed as the mean \pm standard deviation. A Student's t-test was used to analyze the differences between two groups. A 
A

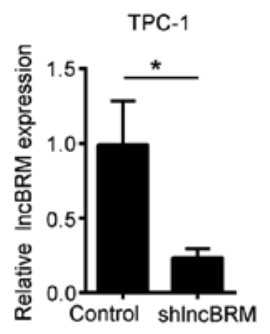

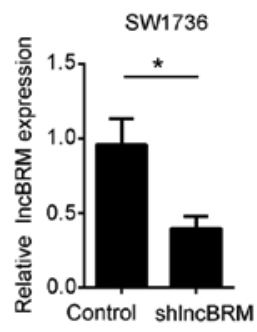

B

TPC-1

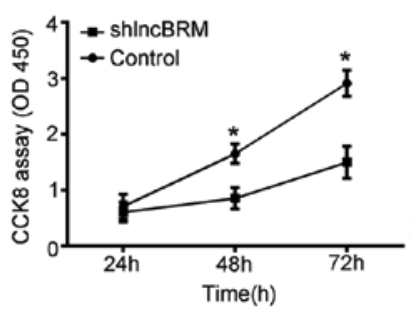

SW1736

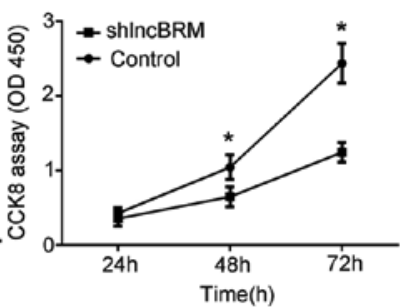

C
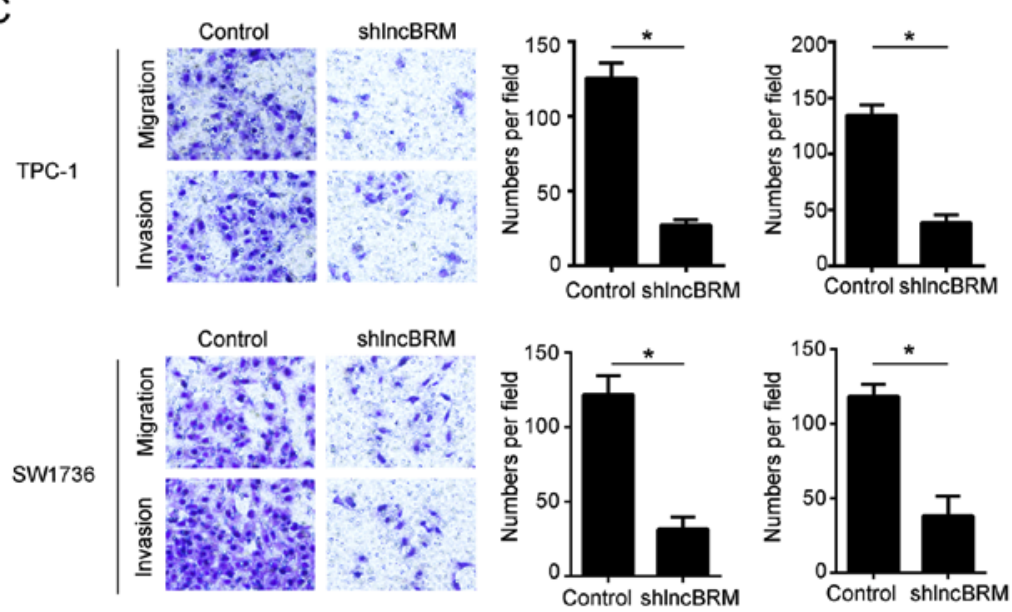

D
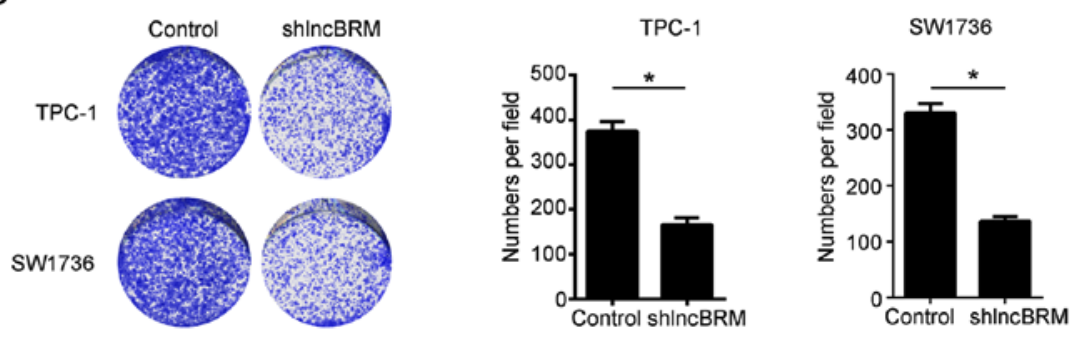

Figure 2. IncBRM knockout impairs the proliferative, migratory and invasive abilities of TPC-1 cells and SW1736 cells. (A) Expression levels of lncBRM were examined by RT-qPCR. Knockout efficiency of IncBRM was determined in TPC-1 and SW1736 cells. Fold changes were normalized to $18 \mathrm{~S}$. (B) Cell Counting Kit- 8 assay was performed to examine the proliferation curves of TPC-1 and SW1736 cells following transfection with the negative control or shlncBRM. (C) Transwell assay was performed to determine the migration and invasive abilities of TPC-1 cells and SW1736 cells, following transfection with the negative control or shlncBRM. (D) Colony-formation assay was performed to determine the proliferative abilities of TPC-1 and SW1736 cells, transfected with negative control or shlncBRM. " $\mathrm{P}<0.05$. RT-qPCR, revere transcription-quantitative PCR; lnc, long non-coding; PTC, papillary thyroid carcinoma; sh, short hairpin.

one-way ANOVA with a pos-hoc Tukey's test was used for multiple comparisons. Kaplan-Meier analysis followed by a log-rank test was used to analyze survival rate. The association between IncBRM expression and clinical features listed in Table I was analyzed using a $\chi^{2}$ test. $\mathrm{P}<0.05$ was considered to indicate a statistically significant difference.

\section{Results}

lncBRM is upregulated in PTC tissues and cell lines. In order to determine the role of IncBRM in PTC progression, RT-qPCR was performed, which revealed increased expression of IncBRM in PTC tissues compared with the adjacent normal tissue ( $\mathrm{P}<0.05$; Fig. 1A). Consistently, lncBRM was also upregulated in the PTC cell lines TPC-1 and SW1736, compared with the normal thyroid Nthy-ori 3-1 cell line (both $\mathrm{P}<0.05$; Fig. 1B). To further examine the prognostic significance of IncBRM, overall survival time was analyzed. Based on the median level of IncBRM expression, the 90 tissues were divided into two groups, lncBRM high and lncBRM low expression groups. Higher expression of $\operatorname{lncBRM}$ was associated with poor overall survival time in patients with PTC $(\mathrm{P}<0.05 ;$ Fig. $1 \mathrm{C})$. Moreover, high expression of IncBRM was associated with metastasis and advanced stages of PTC (both P $<0.05$; Fig. 1D and E). The potential association between IncBRM expression and the clinicopathological features of patients with PTC was also explored. High lncBRM expression was associated with late Tumor-Node-Metastasis $(\mathrm{P}=0.033)$ stages and lymph node metastasis $(\mathrm{P}=0.02$; Table I). Overall, IncBRM was upregulated in PTC and was associated with a poor prognosis.

lncBRM knockout inhibits the progression of PTC cells. In order to elucidate the mechanism by which IncBRM regulates PTC progression, TPC-1 and SW1736 cells were transfected with shRNA targeting IncBRM and expression of lncBRM was significantly decreased in the transfected cells (both $\mathrm{P}<0.05$; Fig. 2A). A CCK-8 assay was performed to examine the proliferation of shcontrol or shlncBRM TPC-1 and SW1736 
A

$\operatorname{IncBRM}(\mathrm{WT})$

miR331-3p 3'-AAGAUCCUAUCCGGGUCCCCG-5'

InCBRM (MUT) 5'-TTCTTCCAAAAAATTGATTTG-3'

C

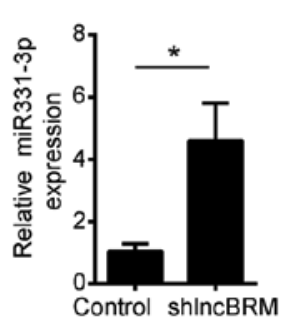

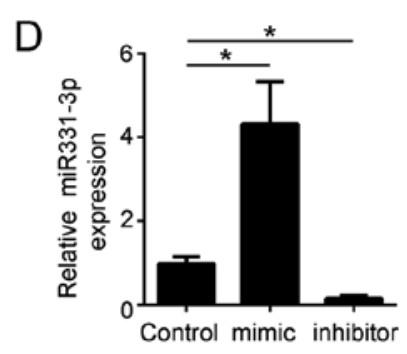

$\mathrm{F}$

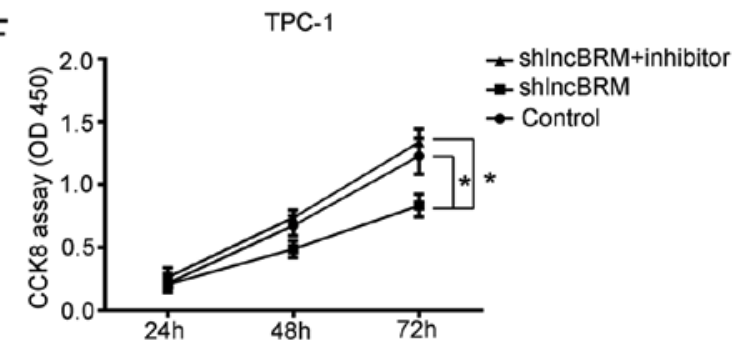

B

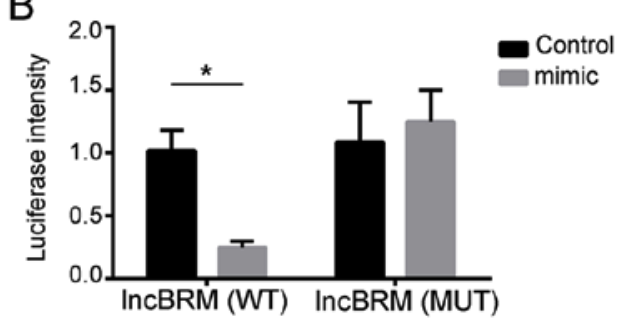

E

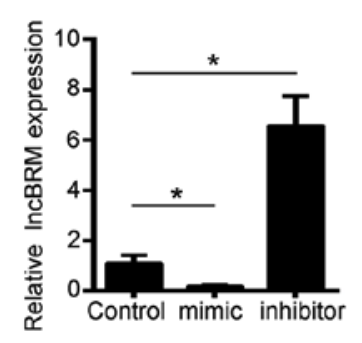

SW1736

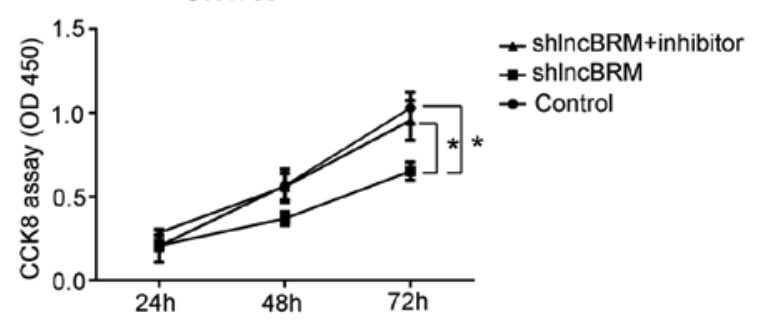

Figure 3. IncBRM interacts with miR-331-3p. (A) Schematic representation of the predicted target site for miR-331-3p and lncBRM. (B) Luciferase reporter assay was performed in TPC-1 cells. Cells were co-transfected with the reporter plasmid (or the corresponding mutant reporter) and the indicated miRs. RT-qPCR was performed to examine the relative expression of miR-331-3p, following transfection with (C) negative control or shlncBRM plasmids and (D) negative control, miR-331-3p mimic or miR-331-3p inhibitor. (E) RT-qPCR was performed to examine the relative expression of lncBRM in cells transfected with negative control or ectopic miR-331-3p expression or inhibition of miR-331-3p. Fold changes were normalized to 18S for all RT-qPCR experiments. (F) Proliferative ability of TPC-1 and SW1736 cells, following transfection with the negative control, shlncBRM alone or with miR inhibitor, were determined through CCK-8 assays. "P<0.05. RT-qPCR, reverse transcription-quantitative PCR; lnc, long non-coding; sh, short hairpin; CCK-8, Cell Counting Kit-8; WT, wild type; MUT, mutant; miR, microRNA.

cells. IncBRM knockout resulted in significantly decreased proliferation of TPC-1 and SW1736 cells after 48 and $72 \mathrm{~h}$ (both $\mathrm{P}<0.05$; Fig. 2B). Transwell assays revealed significantly decreased invasion and migration of TPC-1 cells and SW1736 cells following lncBRM knockout (all $\mathrm{P}<0.05$; Fig. 2C). Furthermore, colony-formation assays revealed that IncBRM promoted the proliferation ability of TPC-1 and SW1736 cells $(\mathrm{P}<0.05$; Fig. 2D).

lncBRM negatively regulates miR-331-3p expression in $P T C$. Bioinformatics analysis was conducted in order to elucidate the mechanism by which IncBRM promotes PTC progression. IncBRM formed complementary base pairing with miR-331-3p (Fig. 3A). To verify whether IncBRM binds to $\mathrm{miR}-331-3 \mathrm{p}$ directly, a luciferase reporter assay was performed. The luciferase activity was significantly decreased following co-transfection with WT-lncBRM and miR-331-3p mimics $(\mathrm{P}<0.05$; Fig. 3B). However, luciferase activity remained unchanged in cells co-transfected with WT-lncBRM and miR-331-3p mimics (Fig. 3B). Moreover, the expression of miR-331-3p was increased following lncBRM knockout compared with the control cells $(\mathrm{P}<0.05 ;$ Fig. $3 \mathrm{C})$. In order to investigate the association between miR-331-3p and lncBRM, cells were transfected with miR-331-3p mimics or inhibitor plasmids and transfection efficiency was confirmed (Fig. 3D). Transfection of miR-331-3p mimic significantly inhibited the expression of lncBRM in TPC-1 cells, whereas transfection with miR-331-3p inhibitor increased lncBRM expression compared with control (both $\mathrm{P}<0.05$; Fig. 3E). Consistently, CCK-8 assays showed proliferation was decreased after IncBRM knockout in TPC-1 and SW1736 cells compared with control after 48 and $72 \mathrm{~h}$ (both $\mathrm{P}<0.05$ ). However, the decreased proliferation caused by IncBRM knockout was reversed by inhibition of miR-331-3p in TPC-1 and SW1736 cells (Fig. 3F). Overall, these data suggest that lncBRM can bind to miR-331-3p and regulate TPC-1 cell proliferation.

IncBRM regulates SLC25A1 expression through miR-331-3p. A TargetScan7 analysis was performed to identify potential genes that are regulated by miR-331-3p, and SLC25A1 was identified as a potential candidate gene. There was a potential binding site at SLC25A1-3'UTR with miR-331-3p (Fig. 4A). The role of SLC25A1 in PTC progression was explored in the present study. Firstly, ectopic expression of miR-331-3p or IncBRM knockout resulted in significantly decreased luciferase activity of wild type $(\mathrm{P}<0.05$; Fig. 4B) but not mutant SLC25A1-3'UTR. Inhibition of miR-331-3p restored the decreased luciferase activity caused by IncBRM knockout 
A
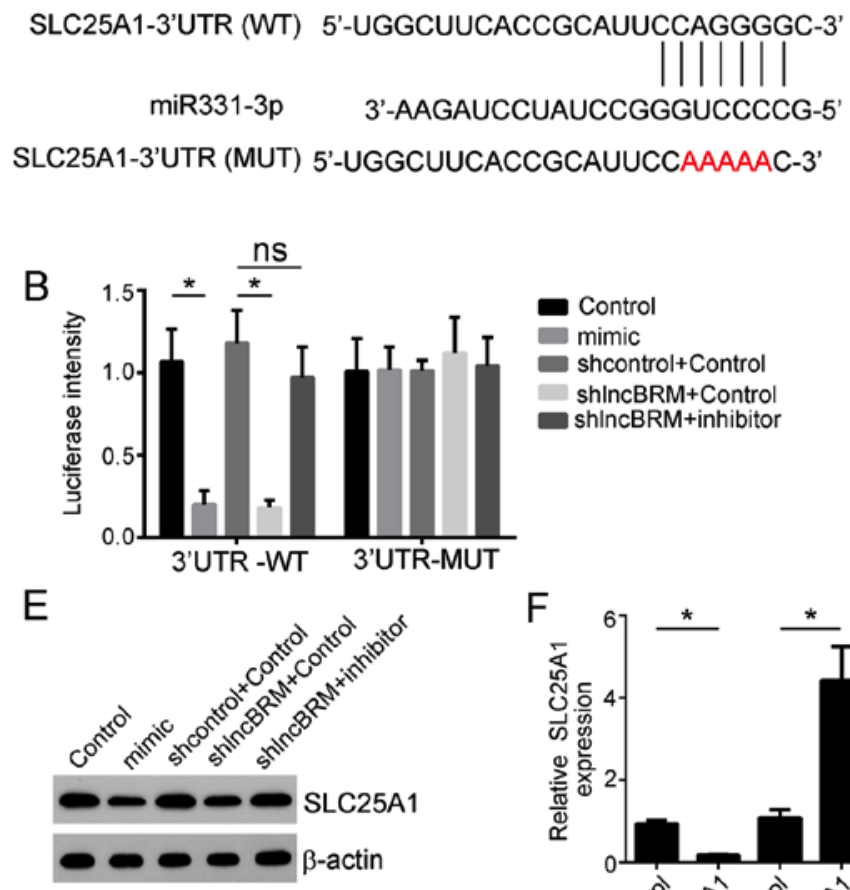

G
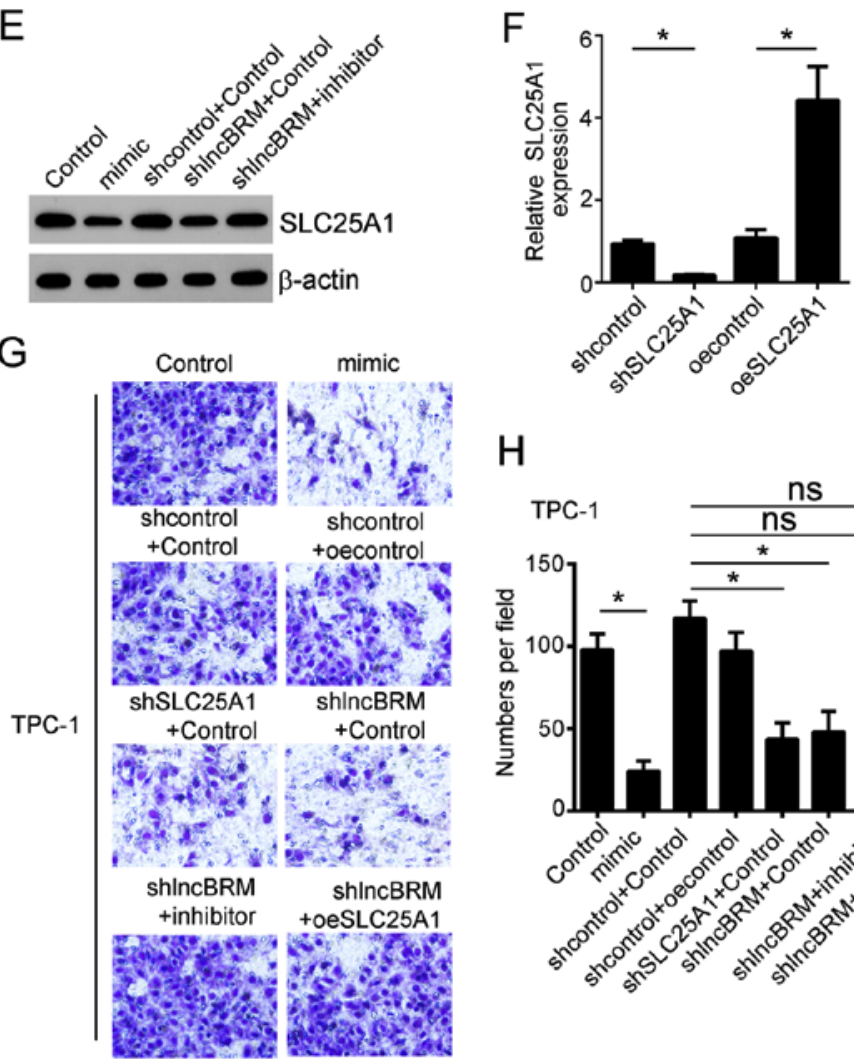

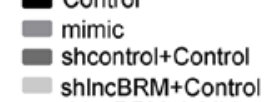

shincBRM+inhibitor

$\mathrm{H}$
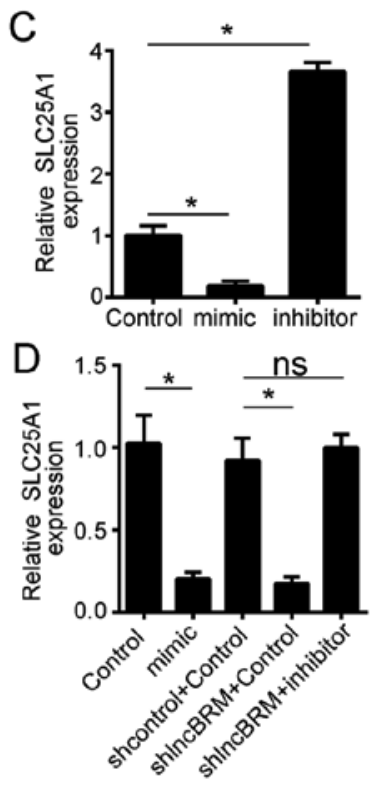
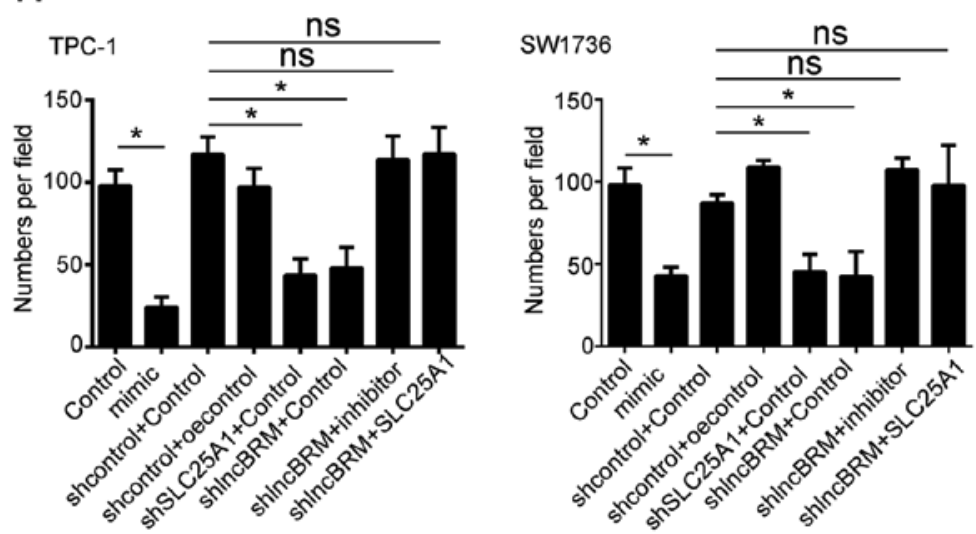

Figure 4. IncBRM regulates PTC progression through sponging miR-331-3p and targeting SLC25A1. (A) Schematic representation of the predicted target site for miR-331-3p and SLC25A1. (B) Luciferase activity assay in the WT or MUT reporter containing SLC25A1 3'UTR when transfected with different vectors in TPC-1 cells. Cells were co-transfected with the reporter plasmid (or the corresponding mutant reporter) and the indicated miRs. The relative expression of SLC25A1 following (C) ectopic miR-331-3p expression or inhibition of miR-331-3p in TPC-1 cells and (D) ectopic miR-331-3p expression or knockout of lncBRM and inhibition of miR-331-3p in TPC-1 cells, examined by RT-qPCR. Samples were normalized to 18S. (E) The protein expression of SLC25A1 was examined by western blot analysis. Samples were normalized to $\beta$-actin. (F) The relative expression of SLC25A1 following knockout or overexpression of SLC25A1. Samples were normalized to 18S. (G and H) Migratory abilities of TPC-1 cells and SW1736 cells transfected with control and different plasmids, examined using transwell assays. "P<0.05. RT-qPCR, revere transcription-quantitative PCR; lnc, long non-coding; sh, short hairpin; WT, wild type; MUT, mutant; miR, microRNA; UTR, untranslated region, NS, not significant.

(Fig. 4B). Ectopic expression of miR-331-3p resulted in decreased SLC25A1 expression ( $\mathrm{P}<0.05)$, whereas miR-331-3p inhibitor promoted SLC25A1 expression ( $\mathrm{P}<0.05$; Fig. 4C). Furthermore, overexpression of miR-331-3p or knockout of lncBRM resulted in decreased SLC25A1 expression, whereas inhibiting miR-331-3p restored this phenotype (all $\mathrm{P}<0.05$; Fig. 4D and E). These data suggest that IncBRM regulates SLC25A1 expression by targeting miR-331-3p.
To determine whether IncBRM exerts its biological effects through miR-331-3p and SLC25A1, rescue assays were performed by overexpressing of SLC25A1 or inhibiting SLC25A1 in lncBRM knockout TPC-1 and SW1736 cells (Fig. 4F). Transwell assays demonstrated that overexpression of miR-331-3p and knockout of lncBRM or SLC25A1 resulted in significantly decreased migration of cells (all $\mathrm{P}<0.05$ ). Inhibition of miR-331-3p or overexpression of SLC25A1 in 
lncBRM-depleted TPC-1 and SW1736 cells significantly restored cell migration abilities (Fig. 4G and $\mathrm{H}$ ).

\section{Discussion}

Thyroid cancer is one of the most prevalent endocrine tumors and the incidence rate is increasing steadily. Therefore, it is important to illustrate the mechanisms of thyroid cancer (31). In recent years, IncRNAs have been shown to serve vital roles in many biological processes (32). Moreover, several studies revealed the roles of lncRNAs in the regulation of PTC progression. For example, Xia et al (33) reported that lncRNA HOXA-AS2 was upregulated in PTC tissues and promoted migration and invasion of PTC cells by regulating the miR-520c-3p/S100A4 pathway. Wang et al (34) demonstrated that LINC01186 suppressed proliferation and invasion of PTC cells by decreasing the expression of YAP1 and increasing LATS1 expression.

lncBRM was found to play vital roles in some cancer types. $\mathrm{Xi}$ et al (27) found that lncBRM facilitated ovarian cancer cell proliferation, migration and invasion via SOX4 upregulation. Li et al (28) reported that IncBRM can sponge miR-204-3p and target TPT1 to promote colorectal cancer cell migration, invasion and proliferation. However, the role of IncBRM in thyroid cancer remains unclear. In the present study, IncBRM was determined to be upregulated in PTC tissues and cell lines. IncBRM knockout decreased TPC-1 cell proliferation, migration and invasion. Furthermore, bioinformatics analysis identified miR-331-3p as a potential target of IncBRM.

Solute carrier (SLC) members are a type of membrane transport proteins (35). SLC25A1 is a mitochondrial carrier, which promotes the flux of citrate/isocitrate across the mitochondria (35). Previously, SLC25A1 was reported to play a vital role in promoting the mitochondrial pool of citrate and redox balance and therefore promoting self-renewal abilities of cancer stem cells in non-small cell lung cancer (36). In addition, SLC25A1 is required for the conversion of glucose into acetyl-CoA for fatty acid synthesis and is increased by PGCl $\alpha$ to promote tumor growth in liver and colon cancer (37). However, the role of SLC25A1 in thyroid cancer remains unclear. In the present study, IncBRM was demonstrated to sponge miR-331-3p and target SLC25A1. The knockout of SLC25A1 resulted in decreased TPC-1 cell migration and invasion, which was rescued by the overexpression of SLC25A1. Thus, SLC25A1 is involved in PTC progression. However, the role of SLC25A1 in other cancer types remains to be explored.

Despite several studies demonstrating the role of lncRNAs in promoting or inhibiting cancer progression (38-40), there are still unanswered questions. For example, most lncRNAs have no coding potential, however, some may regulate cancer progression by coding for small peptides. The ceRNA hypothesis suggests that most lncRNAs are implicated in the pathogenesis of cancer, by serving as miRNA sponges to modulate the expression of miRNA target genes (41). However, whether other mechanisms exist, requires further exploration.

In conclusion, the present study demonstrated increased expression of IncBRM in PTC tissues and cell lines. Bioinformatics analysis predicts IncBRM as a sponge for miR-331-3p that target SLC25A1. Overexpression of IncBRM promotes PTC cells proliferation, migration and invasion, which were rescued by ectopic expression of miR-331-3p or SLC25A1 knockout.

\section{Acknowledgements}

Not applicable.

\section{Funding}

No funding was received.

\section{Availability of data and materials}

All data generated or analyzed during this study are included in the published article.

\section{Authors' contributions}

SL and XH designed the study, analyzed and interpreted the data, and wrote the manuscript. DZ, LC and SG performed some experiments. All authors read and approved the final manuscript.

\section{Ethics approval and consent to participate}

For the use of human samples, the protocol for this study was approved by the Institutional Ethics Committee of The People's Hospital of Tong Liang District and all enrolled patients signed a written informed consent document.

\section{Patient consent for publication}

Not applicable.

\section{Competing interests}

The authors declare that they have no competing interests.

\section{References}

1. Chen C, Huang S, Huang A, Jia Y, Wang J, Mao M, Zhou J and Wang L: Total endoscopic thyroidectomy versus conventional open thyroidectomy in thyroid cancer: A systematic review and meta-analysis. Ther Clin Risk Manag 14: 2349-2361, 2018.

2. Huang F, Zhang Q, Chen W, Zhang H, Lu G, Chen J and Qiu C: Long noncoding RNA cancer susceptibility candidate 2 suppresses papillary thyroid carcinoma growth by inactivating the AKT/ERK1/2 signaling pathway. J Cell Biochem 120: 10380-10390, 2019.

3. Liu J, Wang Y and Zhang B: Advances in diagnosis and treatment of thyroid cancer in children and adolescents. Zhongguo Yi Xue Ke Xue Yuan Xue Bao 40: 838-842, 2018 (In Chinese).

4. Davis PJ, Tang HY, Hercbergs A, Lin HY, Keating KA and Mousa SA: Bioactivity of thyroid hormone analogs at cancer cells. Front Endocrinol (Lausanne) 9: 739, 2018.

5. Albano D, Durmo R, Bertagna F and Giubbini R: $18 \mathrm{~F}$-choline PET/CT incidental thyroid uptake in patients studied for prostate cancer. Endocrine 63: 531-536, 2019.

6. Bolf EL, Sprague BL and Carr FE: A linkage between thyroid and breast cancer: A common etiology? Cancer Epidemiol Biomarkers Prev 28: 643-649, 2018.

7. Enokida T and Tahara M: I. genomic medicine in thyroid cancer. Gan To Kagaku Ryoho 45: 1711-1715, 2018 (In Japanese).

8. Fu XM, Guo W, Li N, Liu HZ, Liu J, Qiu SQ, Zhang Q, Wang LC, $\mathrm{Li} \mathrm{F}$ and $\mathrm{Li} \mathrm{CL}$ : The expression and function of long noncoding RNA IncRNA-ATB in papillary thyroid cancer. Eur Rev Med Pharmacol Sci 21: 3239-3246, 2017. 
9. Guo Y, Zhang P, Sheng Q, Zhao S and Hackett TA: lncRNA expression in the auditory forebrain during postnatal development. Gene 593: 201-216, 2016.

10. Huang F, Chen W, Peng J, Li Y, Zhuang Y,Zhu Z, Shao C, Yang W, Yao $\mathrm{H}$ and Zhang S: lncRNA PVT1 triggers Cyto-protective autophagy and promotes pancreatic ductal adenocarcinoma development via the miR-20a-5p/ULK1 Axis. Mol Cancer 17: 98, 2018.

11. Sun C, Luan S, Zhang G, Wang N, Shao H and Luan C: CEBPA-mediated upregulation of the lncRNA PLIN2 promotes the development of chronic myelogenous leukemia via the GSK3 and Wnt $/ \beta$-catenin signaling pathways. Am J Cancer Res 7: 1054-1067, 2017.

12. Ye B, Liu B, Yang L, Zhu X, Zhang D, Wu W, Zhu P, Wang Y, Wang S, Xia P, et al: $\operatorname{lncKdm} 2 b$ controls self-renewal of embryonic stem cells via activating expression of transcription factor Zbtb3. EMBO J 37: pii: e97174, 2018.

13. Zheng JL, Sun J, Zhang H and Zhang Y: Role of microRNA and lncRNA in lens development and cataract formation. Zhonghua Yan Ke Za Zhi 54: 390-395, 2018 (In Chinese).

14. Guo CJ, Zhang W and Gershwin ME: Long noncoding RNA $1 \mathrm{ncKdm} 2 \mathrm{~b}$ : A critical player in the maintenance of group 3 innate lymphoid cells. Cell Mol Immunol 15: 5-7, 2018.

15. Liu B, Ye B, Yang L, Zhu X, Huang G, Zhu P, Du Y, Wu J, Qin $\mathrm{X}$, et al: Long noncoding RNA lncKdm2b is required for ILC3 maintenance by initiation of Zfp292 expression. Nat Immunol 18: 499-508, 2017.

16. Xiang Y,Huang Y, Sun H,Pan Y, Wu M and Zhang J: Deregulation of miR-520d-3p promotes hepatocellular carcinoma development via 1 ncRNA MIAT regulation and EPHA2 signaling activation. Biomed Pharmacother 109: 1630-1639, 2019.

17. Song Y, Zou L, Li J, Shen ZP, Cai YL and Wu XD: IncRNA SNHG8 promotes the development and chemo-resistance of pancreatic adenocarcinoma. Eur Rev Med Pharmacol Sci 22: 8161-8168, 2018.

18. Yu Y, Shen HM, Fang DM, Meng QJ and Xin YH: lncRNA HCP5 promotes the development of cervical cancer by regulating MACC1 via suppression of microRNA-15a. Eur Rev Med Pharmacol Sci 22: 4812-4819, 2018.

19. Jiang L, Wu Z, Meng $X$, Chu X, Huang $H$ and $X u$ C: lncRNA HOXA-AS2 facilitates tumorigenesis and progression of papillary thyroid cancer through modulating miR-15a-5p/HOXA3 axis. Hum Gene Ther 30: 618-631, 2019.

20. Jiang $\mathrm{W}$, Zhan $\mathrm{H}$, Jiao $\mathrm{Y}$, Li $\mathrm{S}$ and Gao W: A novel lncRNA-miRNA-mRNA network analysis identified the hub lncRNA RP11-159F24.1 in the pathogenesis of papillary thyroid cancer. Cancer Med 7: 6290-6298, 2018.

21. Wang XM, Liu Y, Fan YX, Liu Z, Yuan QL, Jia M, Geng ZS, $\mathrm{Gu} \mathrm{L}$ and $\mathrm{Lu} \mathrm{XB}$ : IncRNA PTCSC3 affects drug resistance of anaplastic thyroid cancer through STAT3/INO80 pathway. Cancer Biol Ther 19: 590-597, 2018.

22. Wu X, Yan Y, Li H, Ji N, Yu T, Huang Y, Shi W, Gao L, Ma L and Hu Y: DNA copy number gain-mediated lncRNA LINC01061 upregulation predicts poor prognosis and promotes papillary thyroid cancer progression. Biochem Biophys Res Commun 503: $1247-1253,2018$

23. Gu Y, Feng C, Liu T, Zhang B and Yang L: The downregulation of lncRNA EMX2OS might independently predict shorter recurrence-free survival of classical papillary thyroid cancer. PLoS One 13: e0209338, 2018

24. Feng L, Yang B and Tang XD: Long noncoding RNA LINC00460 promotes carcinogenesis via sponging miR-613 in papillary thyroid carcinoma. J Cell Physiol 234: 11431-11439, 2019.

25. Zhang $\mathrm{Y}, \mathrm{Hu}$ J, Zhou W and Gao H: lncRNA FOXD2-AS1 accelerates the papillary thyroid cancer progression through regulating the miR-485-5p/KLK7 axis. J Cell Biochem: Nov 19, 2018 (Epub ahead of print). doi: 10.1002/jcb.28072.

26. Zhu P, Wang Y, Wu J, Huang G, Liu B, Ye B, Du Y, Gao G, Tian Y, He L and Fan Z: lncBRM initiates YAP1 signalling activation to drive self-renewal of liver cancer stem cells. Nat Commun 7: 13608, 2016.
27. Xi J, Feng $\mathrm{J}$ and Zeng S: Long noncoding RNA lncBRM facilitates the proliferation, migration and invasion of ovarian cancer cells via upregulation of Sox4. Am J Cancer Res 7: 2180-2189, 2017.

28. Li R, Zhu H, Yang D, Xia J and Zheng Z: Long noncoding RNA lncBRM promotes proliferation and invasion of colorectal cancer by sponging miR-204-3p and upregulating TPT1. Biochem Biophys Res Commun 508: 1259-1263, 2019.

29. DeLellis RA and Williams ED: Tumours of the thyroid and parathyroid. World Health Organization Classification of Tumours. Pathology and Genetics of Endocrine Organs. IARC Press, Lyon, pp 58-70, 2004.

30. Livak KJ and Schmittgen TD: Analysis of relative gene expression data using real-time quantitative PCR and the 2(-Delta Delta C(T)) method. Methods 25: 402-408, 2001.

31. Saini S, Maker AV, Burman KD and Prabhakar BS: Molecular aberrations and alterations in signaling cascades implicated in the pathogenesis of anaplastic thyroid cancer. Biochim Biophys Acta Rev Cancer: Dec 31, 2018 (Epub ahead of print). doi: 10.1016/j. bbcan.2018.12.003.

32. Brajic A, Franckaert D, Burton O, Bornschein S, Calvanese AL, Demeyer S, Cools J, Dooley J, Schlenner S and Liston A: The Long Non-coding RNA Flatr Anticipates Foxp3 expression in regulatory T cells. Front Immunol 9: 1989, 2018.

33. Xia F, Chen Y, Jiang B, Du X, Peng Y, Wang W, Huang W, Feng T and $\mathrm{Li} \mathrm{X}$ : Long noncoding RNA HOXA-AS2 promotes papillary thyroid cancer progression by regulating miR-520c-3p/S100A4 pathway. Cell Physiol Biochem 50: 1659-1672, 2018.

34. Wang N, Duan H, Zhang C, Zhou Y and Gao R: The LINC01186 suppresses cell proliferation and invasion ability in papillary thyroid carcinoma. Oncol Lett 16: 5639-5644, 2018.

35. Palmieri F: The mitochondrial transporter family SLC25: Identification, properties and physiopathology. Mol Aspects Med 34: 465-484, 2013

36. Fernandez HR, Gadre SM, Tan M, Graham GT, Mosaoa R, Ongkeko MS, Kim KA, Riggins RB, Parasido E, Petrini I, et al: The mitochondrial citrate carrier, SLC25A1, drives stemness and therapy resistance in non-small cell lung cancer. Cell Death Differ 25: 1239-1258, 2018.

37. Bhalla K, Hwang BJ, Dewi RE, Ou L, Twaddel W, Fang HB, Vafai SB, Vazquez F, Puigserver P, Boros L and Girnun GD: PGC1 $\alpha$ promotes tumor growth by inducing gene expression programs supporting lipogenesis. Cancer Res 71: 6888-6898, 2011.

38. Chi H, Yang R, Zheng X, Zhang L, Jiang R and Chen J: lncRNA RP11-79H23.3 functions as a competing endogenous RNA to regulate PTEN expression through sponging hsa-miR-107 in the development of bladder cancer. Int J Mol Sci 19: 2531, 2018.

39. Cui S, Yang X, Zhang L, Zhao Y and Yan W: IncRNA MAFG-AS1 promotes the progression of colorectal cancer by sponging miR-147b and activation of NDUFA4. Biochem Biophys Res Commun 506: 251-258, 2018.

40. Hao Y, Yang X, Zhang D, Luo J and Chen R: Long noncoding RNA LINC01186, regulated by TGF-3/SMAD3, inhibits migration and invasion through Epithelial-Mesenchymal-Transition in lung cancer. Gene 608: 1-12, 2017.

41. Long J, Xiong J, Bai Y, Mao J, Lin J, Xu W, Zhang H, Chen S and Zhao H: Construction and Investigation of a lncRNA-associated ceRNA regulatory network in cholangiocarcinoma. Front Oncol 9: 649, 2019.

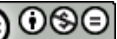

This work is licensed under a Creative Commons Attribution-NonCommercial-NoDerivatives 4.0 International (CC BY-NC-ND 4.0) License. 